\title{
Estado actual del yodo en Colombia y comentarios sobre qué se está haciendo para mejorar la situación del exceso de yodo en el país
}

$\mathrm{S}$ in rodeos, el artículo Análisis del estado poblacional del yodo en Colombia y la necesidad de modificar los niveles de yodación universal de la sal del Dr. Vargas (1) es un excelente artículo, que nos pone en contexto sobre el tema a los que manejamos los asuntos de yodo en el país. Por mi parte, quiero realizar algunos comentarios complementarios a dicho artículo, sobre todo en torno a lo que se está haciendo para mejorar la situación del exceso del yodo en el país.

Para comenzar, quiero relatar que el 6 de junio de 2019, el Ministerio de Salud de Colombia organizó en Bogotá el Encuentro Situación Nacional de Vitaminas y Minerales de Interés en Salud Pública - ENSIN2015 (Encuesta Nacional de la Situación Nutricional 2015), en donde se hizo la presentación oficial de los datos sobre yodo en el país, y se confirmó lo que ya se venía reportando desde hacía varios años atrás: que Colombia es uno de los pocos países del mundo cuyos habitantes presentan excesiva ingesta de yodo, estimado a partir de la elevada mediana en yoduria de varios grupos poblacionales (2). Igualmente, se relievó la importancia de la yodación universal de la sal y de su monitorización continua, y se propusieron soluciones para resolver la situación actual de exceso de yodo, como son la de reducir la norma actual de yodación de la sal, de 50 a 100 ppm, a un nivel menor, 20 a 40 ppm, y la de fortalecer la estrategia de reducción del consumo de sal.

Acerca de la situación de yodo en Colombia reportada en el ENSIN2015 (3), los hallazgos se resumen así:

- En niños de 1 a 4 años ( $n=8097)$, la mediana de yodurias fue de $365 \mu \mathrm{g} / \mathrm{L}$; la prevalencia de exceso de yodo fue del $63,8 \%$ y la de deficiencia de yodo fue del $8,1 \%$.

- En niños de 5 a 12 años ( $n=16161)$, la mediana de yodurias fue de 406,8 $\mu \mathrm{g} / \mathrm{L}$; la prevalencia de exceso de yodo fue del 75,2 \% y la de deficiencia de yodo fue del 4,4 \%.

- En mujeres en edad fértil ( $\mathrm{n}=9828)$, la mediana de yodurias fue de $379 \mu \mathrm{g} / \mathrm{L}$; la prevalencia de exceso de yodo fue del 70,5\% y la de deficiencia de yodo fue del 4,9\%.

La ENSIN2015 también reportó datos de yodurias de acuerdo con el sexo, la etnia, el lugar de residencia (urbano vs. rural), la región del país y el índice de riqueza. En resumen, la deficiencia de yodo fue más prevalente en el área rural y en la población indígena, y el exceso de yodo fue más prevalente en los hombres, en habitantes del área urbana, de la región Atlántica y de índice de riqueza medio.

Los resultados de alta prevalencia de yoduria en los tres grupos estudiados demuestran, por extrapolación, que la población colombiana está expuesta a exceso de yodo. No se midieron las yodurias en embarazadas, pero la alta prevalencia de exceso de yodo en mujeres en edad fértil hace considerar que las mujeres en embarazo puedan tener una buena, e incluso alta, ingesta de yodo. Durante el Encuentro se aclaró, sin embargo, que organizaciones como Iodine Global Network (IGN) y UNICEF no recomiendan que se hable de población con deficiencia o exceso de yodo a partir de los resultados de las yodurias, en tanto que no es posible, con los métodos disponibles actualmente, identificar la proporción de la población con deficiencia de yodo o con ingesta excesiva de yodo (4).

\section{¿Por qué tenemos exceso de yodo en Colombia?}

Colombia es uno de los países con mayor contenido de yodo en la sal para consumo humano (50 a 100 ppm), de acuerdo con la norma establecida por el Ministerio de Salud. Reportes de Refisal, la mayor empresa procesadora, empaquetadora y distribuidora de sal del país, y datos del INVIMA, que hace la monitorización de la sal de esta misma empresa, indican que el contenido de yodo en la sal que ha salido en los últimos tres años de sus procesadoras está entre 64 y 66 ppm, lo cual indica que están cumpliendo con la norma. Además, ha de tenerse en cuenta que el consumo de sal en Colombia oscila entre 10 y 20 gramos, dependiendo de la región y sus costumbres, y del mayor consumo de productos procesados y ultraprocesados. Así, cerca de una quinta parte de la ingesta diaria total de energía de los colombianos procede de productos procesados y ultraprocesados, que bien sabemos son ricos en sal, grasas y azúcares refinados (5). La suma de todos estos factores hace que la población actualmente disponga de mucho yodo. 


\section{Existe preocupación en Colombia por la aparición de "desórdenes por exceso de yodo" (DEY)}

Una manera indirecta de indicar que una población tiene excesivo consumo de yodo es cuando la mediana de yodurias de la población está igual o mayor de $300 \mu \mathrm{g} / \mathrm{L}$. Este corte se tomó porque a partir de este nivel se han registrado algunos trastornos de salud, los desórdenes por exceso de yodo (DEY). Uno de ellos es el aumento de la prevalencia de bocio, algo paradójico pues el bocio es también visto con la deficiencia de yodo. Otro es el incremento de casos nuevos de hipertiroidismo, sobre todo en los primeros años de implementación del programa de yodación de la sal para toda la población. También se aumentó la prevalencia de hipotiroidismo por dos causas: una es por un "bloqueo" que se produce con el exceso de yodo (efecto de Wolff-Chaikoff), pero que generalmente es transitorio, y la otra es por incremento en la prevalencia de las enfermedades autoinmunitarias de tiroides, en parte porque el exceso de yodo vuelve más antigénica a una de las proteínas de la tiroides: la tiroglobulina. Un estudio de Escobar realizado hace 20 años demostró que cerca del $60 \%$ de las personas con bocio, que asistían a su consulta de endocrinología en Bogotá, tenían como causa una tiroiditis autoinmunitaria o de Hashimoto, lo que indicaba que para ese momento ya era causa importante de bocio y de hipotiroidismo en el país (6). Otro estudio publicado hace 30 años, donde fueron reportados 100 casos de tiroiditis de Hashimoto (7), que para la época se sospechaba que los bocios eran ocasionados por deficiencia de yodo o por bociógenos, comenzó a dar indicios de que en Colombia se presentaba dicha tiroiditis, pero que poco se buscaba por falta de sospecha clínica y porque los laboratorios clínicos no procesaban aún los anticuerpos antitiroideos.

Por último, Vargas señala que "una alta ingesta de yodo (especialmente en áreas deficientes) se relaciona con una reducción (no significativa) del riesgo de cáncer tiroideo", pero, por lo contrario, existe la hipótesis de que el exceso de yodo puede elevar los casos nuevos de cáncer papilar de tiroides (CPT). Así, un metaanálisis de 2017 (8) nos indica que en regiones altamente yodinadas existe una asociación positiva entre la exposición al yodo y el CPT (odds ratio [OR]: 2,2; IC 95\%: 1,389-3,483).

\section{Conocido el problema, ¿qué se está haciendo para prevenir o evitar este exceso de yodo?}

Existen dos estrategias para mejorar este estado de exceso de yodo en la población colombiana. La primera es disminuir el contenido de yodo añadido a la sal, es decir, cambiar el Decreto 0547 de 1996. La segunda es tratar de disminuir el consumo de sal de la población.

Sobre la primera medida, actualmente el Ministerio de Salud está llevando a cabo un plan de trabajo que deberá conducir finalmente a un cambio del decreto mencionado. Para esto, elaboró un "Análisis de Impacto Normativo", relacionado con la sal para consumo humano y su contenido de yodo (9), que luego fue llevado a consulta pública nacional, y actualmente está realizando los ajustes del documento, acordes con dicha consulta. Sin embargo, todavía aún se requieren varios pasos de consultas técnicas y jurídicas, nacionales e internacionales, antes de hacer el cambio de la norma de la yodación de la sal, la cual se pretende que quede con 20 a 40 ppm.

Sobre la segunda medida, ya existe en el Ministerio de Salud un programa que busca que los colombianos reduzcamos el consumo de sal a 5 gramos al día, como una medida para disminuir enfermedades cardiovasculares y la hipertensión arterial. La intención ahora es reforzar dicha estrategia para que, al reducir el consumo de sal, también reduzcamos el consumo de yodo contenido en este. Es importante recalcar, como lo resaltó Vargas en su artículo, que la estrategia de reducción del consumo de sal es totalmente compatible con la de yodación de la sal, teniendo en cuenta que los ajustes de la concentración del yodo en la sal deben estar basados en los datos de consumo de sal en la dieta por parte de la población.

Estas dos medidas deberán estar enmarcadas en una estrategia de "educación, información y comunicación", para que se conozca más sobre el tema y nos dispongamos a prevenir tanto la deficiencia como el exceso de yodo, y sus consecuencias. Para lograr esto, son protagónicas las entidades nacionales e internacionales de salud que mantienen vigilancia sobre el yodo, los medios de comunicación masiva y las sociedades científicas, en especial la Asociación Colombiana de Endocrinología, Diabetes y Metabolismo, que es la receptora de este tema por su incumbencia con la tiroides y sus patologías. Igualmente, desde el IGN estamos pendientes de la situación de Colombia, para lo cual se ha hecho un reporte reciente en su Newsletter (10), y seguiremos apoyando y asesorando a las instituciones de salud nacionales que tienen que ver con el control y la vigilancia del yodo.

Iván Darío Escobar, MD

Expresidente, Asociación Colombiana de Endocrinología, Diabetes y Metabolismo. Representante en Colombia, Iodine

Global Network. 


\section{Referencias}

1. Vargas Uricoechea H, Murillo Palacios J, Ramírez Bejarano LE. Análisis del estado poblacional del yodo en Colombia y la necesidad de modificar los niveles de yodación universal de la sal. Revista ACE. 2020;7(2):87-99.

2. Pretell EA. Eliminación sostenible de los desórdenes por deficiencia de yodo en Latinoamérica. Reporte de los talleres subregionales para evaluar la situación de los países de Latinoamérica. IGN/UNICEF/OPS; 2014. p. 3-40

3. Ministerio de Salud de Colombia. Encuesta Nacional de la Situación Nutricional - ENSIN 2015 [Internet]. Disponible en: www.ensin.gov.co

4. Unicef. Guidance on the Monitoring of Salt Iodization Programmes and Determination of Population Iodine Status [Internet]. Disponible en: https:// www.unicef.org/nutrition/files/Monitoring-of-Salt-Iodization.pdf

5. Parra DC, da Costa-Louzada ML, Moubarac JC, Bertazzi-Levy R, Khandpur $\mathrm{N}$, Cediel G, et al. The association between ultra-processed food consumption and the nutrient profile of the Colombian diet in 2005. Salud Publica Mex. 2019;61(2):147-54. doi: 10.21149/9038
6. Escobar ID. Estudio de pacientes con bocio en una consulta endocrinológica. En: Libro de resúmenes del VIII Congreso Latinoamericano de Tiroides de la SLAT. Brasil: Sociedad Latinoamericana de Tiroides; 27 a 30 de mayo de 1999. p. 100.

7. Escobar ID, Kattah W, Niño A, Acosta E, Saavedra C, Ucrós A. Tiroiditis de Hashimoto. Estudio de 100 casos. Acta Med Col. 1991;16(1):18-29. Disponible en: https://bit.ly/31dJ26V.

8. Lee JH, Hwang Y, Song RY, Yi JW, Yu HW, Kim SJ, et al. Relationship between iodine levels and papillary thyroid carcinoma: A systematic review and meta-analysis. Head Neck. 2017;39(8):1711-18. doi: 10.1002/hed.24797

9. Subdirección de Salud Nutricional Alimentos y Bebidas. Impacto normativo relacionado con sal para consumo humano [Internet]. Ministerio de Salud y Protección Social; 2019. Disponible en: https://bit.ly/3guG7gA

10. Escobar ID, Higa AM. To prevent iodine excess, Colombia plans to lower the iodine content of salt while emphasizing population salt reduction. IDD Newsletter Iodine Global Network. 2019;47(3):16-17. Disponible en: https://bit.ly/2PlQCXy. 


\section{Synthroid 0}

\section{PALPAR EL CUELLO Y EVALUAR LA TSH, PUEDE ALERTAR SOBRE ALGÚN TRASTORNO TIROIDEO ${ }^{(1,23)}$}

\section{CUELLOS}

QUE CAUTIVAN...

MARCA DE REFERENCIA EN COLOMBIA

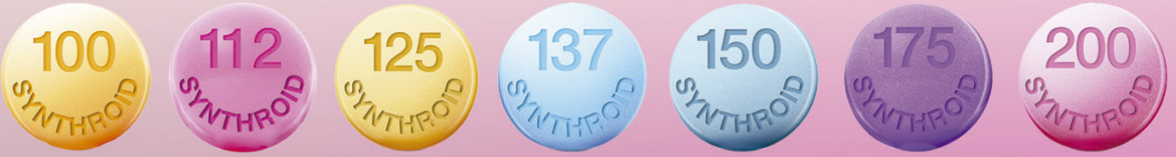

Synthroid ${ }^{\oplus}$

$(((0))$

Levotiroxina sódica
$137 \mathrm{mcg}$
60 Tabletas $-V_{\text {Va }}$ Oral

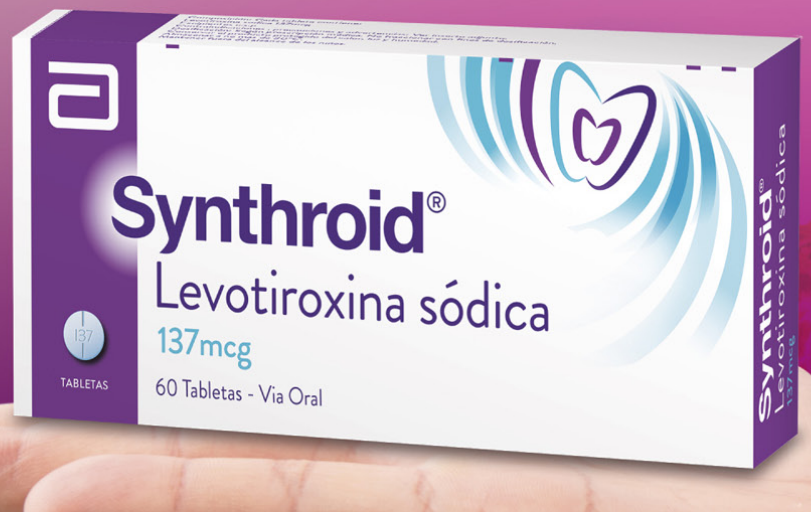

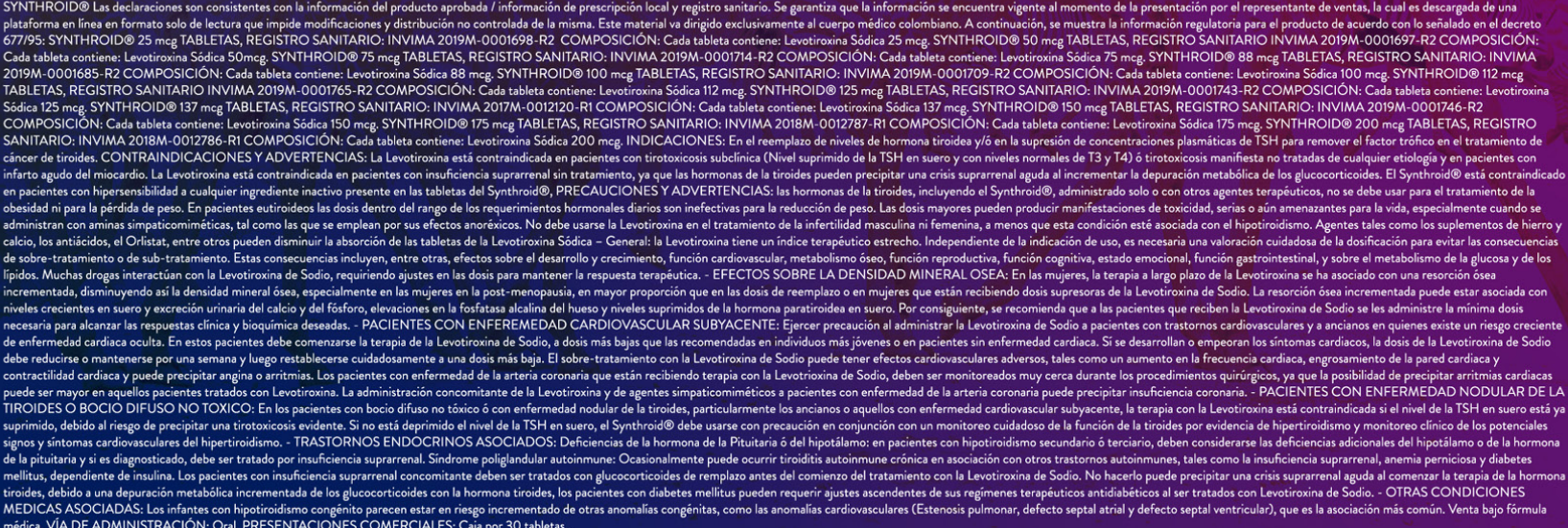

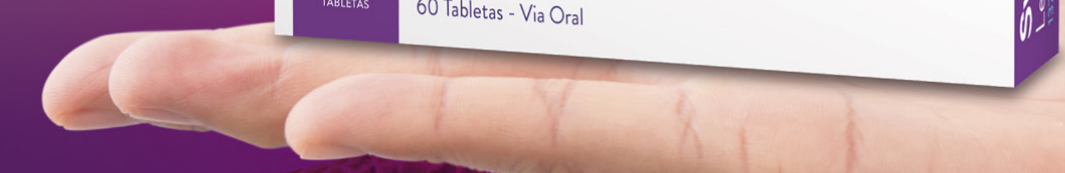

